\title{
Early intervention, treatment and rehabilitation of employees with common mental disorders by using psychotherapeutic consultation at work: study protocol of a randomised controlled multicentre trial (friaa project)
}

Jeannette Weber ${ }^{1 *}$ (D) Peter Angerer ${ }^{1}$, Lorena Brenner ${ }^{2}$, Jolanda Brezinski ${ }^{3}$, Sophia Chrysanthou ${ }^{2}$, Yesim Erim $^{4}$, Manuel Feißt ${ }^{3}$, Marieke Hansmann ${ }^{5}$, Sinja Hondong ${ }^{4}$, Franziska Maria Kessemeier ${ }^{6}$, Reinhold Kilian ${ }^{7}$, Christina Klose ${ }^{3}$, Volker Köllner ${ }^{2}$, Fiona Kohl ${ }^{1}$, Regina Krisam³ ${ }^{3}$, Christoph Kröger ${ }^{5}$, Anja Sander ${ }^{3}$, Ute Beate Schröder ${ }^{8}$, Ralf Stegmann ${ }^{8}$, Uta Wegewitz ${ }^{8}$, Harald Gündel ${ }^{9}$, Eva Rothermund ${ }^{9}$ and Kristin Herrmann ${ }^{9}$

\begin{abstract}
Background: Common mental disorders are one of the leading causes for sickness absence and early retirement due to reduced health. Furthermore, a treatment gap for common mental disorders has been described worldwide. Within this study, psychotherapeutic consultation at work defined as a tailored, module-based and work-related psychotherapeutic intervention will be applied to improve mental health care.

Methods: This study comprises a randomised controlled multicentre trial with 1:1 allocation to an intervention and control group. In total, 520 employees with common mental disorders shall be recruited from companies being located around five study centres in Germany. Besides care as usual, the intervention group will receive up to 17 sessions of psychotherapy. The first session will include basics diagnostics and medical indication of treatment and the second session will include workrelated diagnostics. Then, participants of the intervention group may receive work-related psychotherapeutic consultation for up to ten sessions. Further psychotherapeutic consultation during return to work for up to five sessions will be offered where appropriate. The control group will receive care as usual and the first intervention session of basic diagnostics and medical indication of treatment. After enrolment to the study, participants will be followed up after nine (first follow-up) and fifteen (second follow-up) months. Self-reported days of sickness absence within the last 6 months at the second follow-up will be used as the primary outcome and self-efficacy at the second follow-up as the secondary outcome. Furthermore, a costbenefit assessment related to costs of common mental disorders for social insurances and companies will be performed.
\end{abstract}

\footnotetext{
* Correspondence: Jeannette.Weber@hhu.de

${ }^{1}$ Institute of Occupational, Social and Environmental Medicine, Centre for Health and Society, Medical Faculty, Heinrich-Heine-University Düsseldorf, Moorenstraße 5, 40225 Düsseldorf, Germany

Full list of author information is available at the end of the article
}

(c) The Author(s). 2021 Open Access This article is licensed under a Creative Commons Attribution 4.0 International License, which permits use, sharing, adaptation, distribution and reproduction in any medium or format, as long as you give appropriate credit to the original author(s) and the source, provide a link to the Creative Commons licence, and indicate if changes were made. The images or other third party material in this article are included in the article's Creative Commons. licence, unless indicated otherwise in a credit line to the material. If material is not included in the article's Creative Commons licence and your intended use is not permitted by statutory regulation or exceeds the permitted use, you will need to obtain permission directly from the copyright holder. To view a copy of this licence, visit http://creativecommons.org/licenses/by/4.0/ The Creative Commons Public Domain Dedication waiver (http://creativecommons.org/publicdomain/zero/1.0/) applies to the data made available in this article, unless otherwise stated in a credit line to the data. 
Discussion: Psychotherapeutic consultation at work represents a low threshold care model aiming to overcome treatment gaps for employees with common mental disorders. If successfully implemented and evaluated, it might serve as a role model to the care of employees with common mental disorders and might be adopted in standard care in cooperation with sickness and pension insurances in Germany.

Trial registration: The friaa project was registered at the German Clinical Trial Register (DRKS) at 01.03.2021 (DRKS00023049): https:/www.drks.de/drks_web/navigate.do?navigationld=trial.HTML\&TRIAL_ID=DRKS00023049.

Keywords: Mental health, Workplace, Return to work, Psychotherapy, Depression, Self-efficacy, Sickness absence

\section{Background}

Common mental disorders (CMDs) are one of the major public health problems worldwide with an estimated twelve-month global prevalence rate of $17.6 \%$ [1]. According to their high burden of disease [2], CMDs are one of the leading causes for sickness absence [3] and go along with high risks of early retirement [4]. Prevention, early treatment and well-concerted reintegration after sickness absence might act as key factors to reduce the risk of chronicity of CMDs, long and recurrent sickness absence and early retirement due to CMDs. However, even in high-income countries, large treatment gaps were reported. For example, only $5 \%$ of individuals with anxiety disorders and $22 \%$ of individuals with major depressive disorders are estimated to receive adequate treatment $[5,6]$. On the one hand, reasons for this treatment gap are supposed to be person-related including lack of perceived need for treatment [5] and fear of stigmatisation $[7,8]$. On the other hand, reasons are related to the health-care system itself including long waiting times on therapy [9-11].

Psychotherapeutic consultation at work is a new and low threshold concept for early prevention and treatment of employees with symptoms of CMDs [12, 13]. It might offer first consultation, diagnostic identification, referral to care as usual (CAU), proceeding psychotherapy, and therefore possibilities to tackle treatment gaps regarding mental health [14]. First evidence suggests that psychotherapeutic consultation at work significantly reduces depressive symptoms and anxiety and improves work ability [15]. In addition, extrapolation suggests that screening of CMDs and subsequent psychotherapeutic treatment at work is cost effective [16]. Furthermore, psychotherapeutic consultation at work can serve as a model for close collaboration between occupational health physicians, psychotherapists and other mental health care providers [17]. Such collaboration has been regarded as an additional key factor to improve treatment and return to work (RTW) of employees with CMDs [18]. For example, within a previous randomised controlled trial (RCT), referral of employees with depression to psychiatric care by occupational health physicians was combined with collaboration between those health care providers during vocational reintegration. This intervention was found to be associated with faster RTW compared to CAU [19]. More successful and sustainable RTW might further be achieved by combining CAU and work-related psychotherapy as evidenced by previous research [20-26]. Concepts of work-related psychotherapy were most often developed to support vocational reintegration of employees being sick-listed due to CMDs, including - inter alia - work-related assessments, assistance in drawing RTW plans and contacting employers and occupational physicians as well as evaluation of RTW steps [19-24]. However, work-related psychotherapy could also contribute to reduction of depressive symptoms and improvement of work ability at earlier stages [27, 28].

Particularly a combination of a) prevention, b) early treatment, c) work-related psychotherapy and d) collaboration between key (mental) health care professionals might help to tackle the reported treatment gap and therefore chronicity, long and recurrent sickness absence and early retirement due to CMDs. However, within occupational settings, previous studies have mainly investigated the effectiveness of interventions addressing either one or two of those aspects (e.g. [15, 21, 22, 24, 27, 28]). Furthermore, study samples were often small including only one or two companies and cost-benefit assessments are seldom performed (e.g. $[15,19,25,27,28])$. RCTs testing the effectiveness of more comprehensive care models in small, middle as well as large-sized companies are scarce.

This two-arm randomised controlled multicentre trial - called early intervention in the workplace (German: Frühe Intervention am Arbeitsplatz, friaa) - therefore aims to evaluate psychotherapeutic consultation at work, which in this study consists of a tailored, module-based and work-related psychotherapeutic intervention. The intervention will thereby combine the four aspects of prevention, early treatment, work-related psychotherapy and collaboration between key (mental) health care professionals. Employees with symptoms of CMDs will be randomised with 1:1 allocation to an intervention group receiving psychotherapeutic consultation at work or to a control group receiving CAU. Participants' outcome data 
of both groups will be collected nine (first follow-up) and fifteen (second follow-up) months after enrolment.

The primary aim is to investigate whether the intervention is superior to CAU regarding reduction of sickness absence within the last 6 months at the second follow-up among employees with symptoms of CMDs.

The secondary aims are to investigate whether the intervention is superior to CAU regarding

- levels of occupational self-efficacy at the second follow-up among employees with symptoms of CMDs

- a health economic evaluation related to costs of CMDs for social insurances and companies at second follow-up

\section{Methods/design}

Participants, interventions, and study variables

\section{Study setting and recruitment}

Participants will be recruited from small, middle and largesized companies being located around five study centres in Germany (in and around Ulm, Düsseldorf, Teltow, Hildesheim and Erlangen) over a period of twelve months, starting in September 2021. Psychotherapeutic consultation at work will be established in close collaboration with occupational health services in or outside of business premises based on preferences of participating companies. Employees with psychological complaints will then be transferred to psychotherapeutic consultation at work by occupational health physicians, supervisors or by selfassignment. Each employee who is transferred to psychotherapeutic consultation at work will receive diagnostic assessment regarding mental health and recommendations for further procedures in CAU (Module 1-A). They will be screened for eligibility criteria and if eligible, will be asked to participate by written informed consent. Participants will then be randomised to the intervention group or control group with 1:1 allocation. Participants not meeting the eligibility criteria will get the according recommendation to CAU. The intervention modules will be accompanied by setting up a network of occupational health services, psychotherapists conducting the psychotherapeutic consultation, clinics for psychosomatic medicine and rehabilitation centres depending on local conditions. Communication between those (mental) health care providers will be established in the form of standardised handovers. Furthermore, educational programs will be offered to all occupational physicians and psychotherapists taking part in the intervention.

\section{Eligibility criteria}

Inclusion criteria:

- written informed consent to participate in the study
- aged 18 years or above

- sufficient knowledge of the German language to participate in the study

- employment for at least $15 \mathrm{~h}$ per week in participating companies

- diagnosis of a CMD according to the International Statistical Classification of Diseases and Related Health Problems (ICD-10 [29]) including unipolar depressive disorders (F32-F34), anxiety disorders (F40, F41), stress-related and somatoform disorders (F42, F43, F45, F48.0) and non-organic sleep disorders (F51) or subclinical symptoms of psychosomatic disorders without ICD-10 diagnosis

- symptoms of psychosomatic disorders without ICD10 diagnosis measured by the Global Assessment of Functioning Scale (GAF Scale < 81, [30, 31]).

\section{Exclusion criteria:}

- unique or main diagnosis of substance abuse (F10-19), schizophrenia, psychosis (F20-F29) or organic psychiatric disorders (F00-F09)

- severe and unstable somatic health condition (e.g. cancer)

- current psychotherapeutic treatment

- application for retirement pension

\section{Intervention}

All sessions of psychotherapeutic consultation at work will be conducted by licenced psychological or medical psychotherapists or by psychological or medical psychotherapists being in an advanced state of postgraduate training for psychotherapy. The intervention consists of three modules. The first module includes one session of basic diagnostic assessment on mental health (Module 1-A) and one session of work-related diagnostic assessment (Module 1-B). Whereas Module 1-A will be offered to the intervention and control group, Module 1-B and all other modules will exclusively be offered to the intervention group. Based on mental health assessment, participants of the control group will receive recommendations for treatment in CAU (e.g. in- or outpatient acute care, inpatient vocational rehabilitation). Participants of the intervention group will be transferred to CAU if outpatient work-related treatment would not sufficiently cover the individual need for treatment or will receive Module 2. In case participants of the intervention group are transferred to CAU, potential waiting times for treatment will be bridged by Module 2. Module 2 will include up to ten sessions of work-related psychotherapeutic consultation or treatment. Participants of the intervention group with an inability to work will receive further psychotherapeutic consultation during their RTW process in up to five sessions during Module 3. 
Participants of the intervention group may thus receive up to 17 sessions of psychotherapeutic consultation (Module 1-A: one session; Module 1-B: one session; Module 2: ten sessions; Module 3: five sessions). For both, the control and intervention group, a medical report will be prepared. Participants of the intervention group who received up to four sessions of psychotherapeutic consultation and participants of the control group will receive a follow-up call by study therapists twelve weeks after their last visit. In this call, participants will be asked on how they have coped so far and whether further help regarding treatment in CAU is needed. For all other participants, an equal follow-up will be performed during their last session of psychotherapeutic consultation.

A schematic overview of the intervention is given in Fig. 1 and all its components are further described below.

\section{Module 1-a. basic clinical assessment}

- Medical and psychosomatic history

- International Neuropsychiatric Interviews (MINI [32])

- Screening of eligibility and inclusion in the study

- Randomisation of participants into intervention or control group

- Recommendations for further procedures in CAU (e.g. in- or outpatient acute care, inpatient vocational rehabilitation)

- Participants of the intervention group may further be referred to the second module of the intervention.

\section{Module 1-B. work-related assessment}

- Work-related assessment including work history, job description, work tasks, work relationships and person-job fit as described elsewhere [25, 33]. If possible, information on those aspects being provided by occupational physicians will also be taken into account

- Analysis of working conditions, social conflicts and social-therapeutic aspects including general social problems or concealed resources as described by Köllner [34]

Module 2. Work-related psychotherapeutic intervention During module 2, an adaption of the manual from Bode et al. [33] will be used to conduct work-related psychotherapeutic consultation. This will include

- Psychoeducation providing information on the relations between work and mental health

- Formation of an individual exploratory model of current psychosomatic symptoms. In this model, stressors and resources at work will be included
- Using communication techniques to increase, maintain or restore motivation to work

- Defining achievable tasks as well as focusing on achievements and resources at work to promote self-efficacy

- Discussing problems at work, elaboration of solution approaches and searching for contact persons at work to implement those solution approaches

- Evaluation and treatment of work-related symptoms (e.g. anxiety or overload) by using cognitive restructuring methods

- Implementation of work-related cognitivebehavioural therapeutic treatment measures: resource activating measures, relaxation techniques that can be used at the workplace, coaching of social competences, communication exercises, acceptance of unchangeable situations, clarification of intrapersonal conflicts, promotion of acceptance and emotional competence

- Detection of work-related risk factors for development and deterioration of CMDs (e.g. shift work) and giving recommendations of setting-based prevention measures to minimise them

Depending on severity of symptoms, psychotherapeutic consultation during module 2 may either include more curative or preventive aspects. Therefore, module 2 is subdivided into module 2-A comprising work-related short-term psychotherapy and module 2B comprising work-related prevention. Module 2-A contains both disorder specific psychotherapeutic interventions and the above mentioned work-related interventions. Participants with an ICD-10 diagnosis will thus receive module 2-A and participants with subclinical psychosomatic symptoms without an ICD10 diagnosis will receive module 2-B.

Module 3. Psychotherapeutic consultation during RTW RTW should be prepared and implemented in consideration of the four phase model of vocational reintegration [35]. Therapeutic sessions of module 3 will be offered to participants with prior sickness absence to support RTW. This will include the implementation of therapeutic issues and strategies from module $1-\mathrm{B}$ and 2. Furthermore, module 3 will comprise

- Psychotherapeutic support and evaluation of each reintegration step (e.g. discussion of successful and unsuccessful aspects or needs for adjustments of the reintegration plan) as described by Bode et al. [33]

- If the patient provides informed consent, study psychotherapists will be involved within the occupational process of reintegration and will 

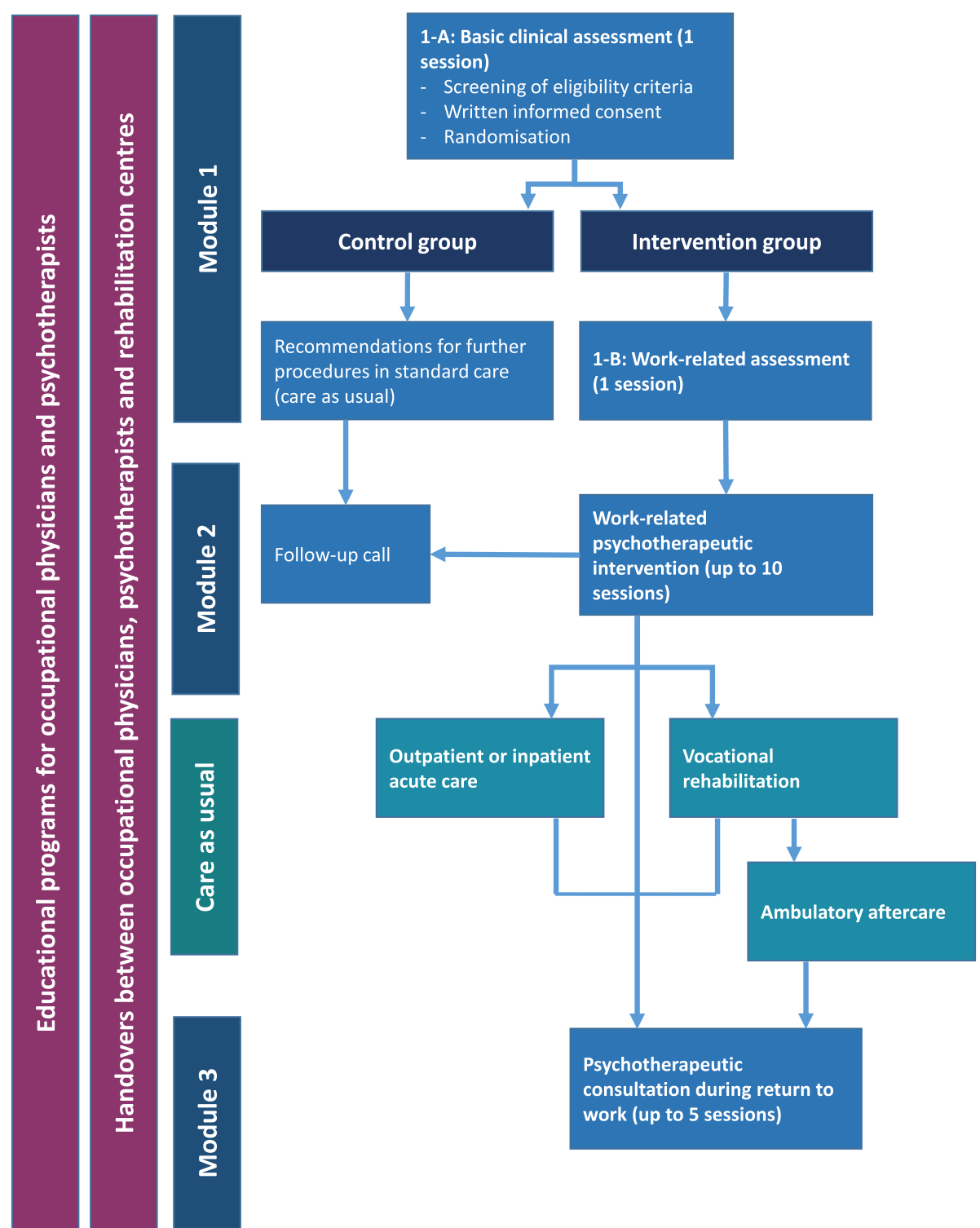

Fig. 1 Overview on study intervention

collaborate with responsible RTW actors (e.g. occupational physician)

Weekly sessions will be intended.

\section{Handovers}

- Accompanying all modules of the intervention

- Standardised forms for communication between occupational physicians, psychotherapists and other care providers

- Handovers will only be performed with patient's permission under consideration of medical confidentiality and data protection legislation
Contents of educational programs for psychotherapists and occupational physicians

- Applications for rehabilitation measures in the German health care system

- Draft of medical reports to increase efficient communication between health care providers

- Information on aims, processes and regulations of prevention and rehabilitation offers of sickness and pension insurances in Germany

- Culture-specific approaches during treatment according to Erim et al. [36]

- Additional contents within the educational program for psychotherapists: training in the intervention 
modules and basic knowledge about occupational health care

- Additional contents within the educational program for occupational health physicians:

Psychotherapeutic primary care

\section{Discontinuation and modifications of the intervention protocol}

The intervention might be discontinued if symptoms decrease and there is no indication for further treatment. If an appointment has been missed, efforts will be made to contact the participant to schedule a new appointment. However, in case that a participant refuses further treatment, the intervention will be discontinued and participants will be asked for their reasons for refusal. If symptoms increase, the psychotherapist and possibly supervisor (i.e. a versed psychotherapist) will weight up further treatment needs. They might schedule a higher frequency of therapy sessions - but no more than 10 sessions of module 2 and 5 sessions of module 3 - or might refer participants to inpatient care. In each case, participants will remain in the study and will be reminded of the follow-up measurements in their last therapy session.

If participants withdraw their informed consent to participate in the study, further therapy sessions will be cancelled. In this case, follow-up measurements will not be performed. If participants also withdraw their informed consent of data storage, collected data of those participants will be deleted and not be included within data analysis.

In general, concomitant care and interventions (e.g. outpatient psychiatric or neurological treatment, somatic treatment, non-medical practitioner treatment, psychological coaching, use of helplines and helpdesks at work) are not prohibited during the trial. Moreover, module 2 aims to bridge waiting times on CAU and therefore also outpatient psychotherapeutic and inpatient psychotherapeutic, psychiatric or rehabilitation treatment are not prohibited during the trial. However, the intervention will be terminated or paused during CAU. Employees receiving psychotherapeutic treatment before starting the intervention are excluded from the study.

In general, discontinuation and all deviations of the intervention protocol will be documented.

\section{Adherence to the study protocol}

Several strategies will be implemented to ensure adherence to the intervention protocol. First, psychotherapists will be trained in the intervention modules during the two-day educational program. Second, psychotherapists will be constantly supervised by a versed therapist and a scientific assistant from the study. Third, therapists will be instructed to record defined treatment sessions on video. The manual from Bode et al. (2017) served as a basis for selecting important interventions [33]. For the evaluation of treatment integrity only those treatment sessions that contain the interventions will be recorded and analysed. Assignment to intervention modules, number of sessions in each module and session dates will be documented and completeness of data will be constantly monitored during the intervention by the psychotherapists and scientific assistants. Furthermore, participants will be regularly reminded of follow-up measurements.

\section{Outcomes}

Primary outcome Days of sickness absence within the last 6 months at second follow-up will serve as the primary outcome. As in comparable previous studies $[25,37]$, days of sickness absence is chosen as the primary outcome due to its economic value and because it is a relevant parameter of psychotherapeutic and psychiatric rehabilitation measures. Days of sickness absence within the last 6 months will be collected as a self-reported item by standardised paper-and-pencil or online questionnaires during baseline and during followups. This item was self-developed for this study. Participants will be asked whether they were absent from work for health reasons within the last 6 weeks with response options "yes" and "no". If participants ticked "yes", they will be asked about the number of days of sickness absence within the last 6 months excluding regular nonworking days.

Main secondary outcomes Self-efficacy at the second follow-up will serve as a secondary outcome. Selfefficacy is chosen as a secondary outcome due to its predictive value of successful RTW [38-40]. Information on self-efficacy will be collected by standardised paper-andpencil or online questionnaires during baseline and during follow-ups.

To measure self-efficacy regarding RTW, a German version of the validated return-to-work self-efficacy scale (RTW-SE [41]) will be used. The RTW-SE scale consists of eleven items with a six-point rating scale from $1=$ "totally disagree" to 6 = "totally agree". Example items are "I will be able to cope with setbacks" and "I will be able to concentrate on my work".

Occupational self-efficacy will be measured by the German version of the validated short form of the Occupational Self-Efficacy Scale [42]. The Occupational SelfEfficacy scale consists of six items with a six-point rating scale from $1=$ "not at all true" to $6=$ "completely true". Example items are "I can remain calm when facing difficulties in my job because I can rely on my abilities" and 
"Whatever comes my way in my job, I can usually handle it".

Both scales were shown to be reliable by previous research with good to excellent internal consistencies $[41,42]$. Therefore, mean scores will be calculated for both scales.

Health economic evaluation Health economic evaluation will be conducted by means of an incremental cost-utility analysis (ICUA) following the net benefit approach [43, 44] from societal perspective [45]. Incremental cost utility ratios (ICUR) will be estimated for estimating the maximum willingness to pay (MWTP) needed for the gain of one quality adjusted life year (QALY) by providing the intervention in comparison to CAU [43, 44]. Comprehensive assessment of health service use and costs will be performed by means of the German version of the client sociodemographic and service receipt inventory (CSSRI $[46,47])$ adjusted for CMDs. QALYs will be estimated by means of the EuroQol (EQ5D-5L [48]) using the German value set $[49,50]$.

\section{Additional data}

Additional data will be collected either by standardised interviews conducted by the study psychotherapists or by standardised paper-and-pencil or online questionnaires. For most variables previously published scales will be used. An overview of additional study variables and relevant references is given in Table 1 . Scales not being previously published (i.e. days of sickness absence and sociodemographic variables) are provided as supplemental material.

\section{Participant timeline}

The schedule of the study including enrolment, intervention and data assessment is given in Table 2.

\section{Diversity issues}

In order to meet the ethnic diversity of the insured, the migration background will be documented and analysed as a sociodemographic variable. In addition, the degree of cultural adjustment and post-migratory stress will be assessed with specialized questionnaires [53, 60, 61]. Since studies have shown that migrants benefit less from psychotherapy than natives [72], training (and supervision if needed) regarding culturally sensitive methods will be provided to optimize outcomes for subjects with a migrant background.

\section{Assignment of interventions Allocation}

Eligible participants will be allocated concealed by randomisation using a centralized web based tool (www. randomizer.at) after Module 1-A. Stratification by centre, days of sickness absence within the last 6 months $(<21$ days vs. $\geq 21$ days $)$ and level of depression measured by the Patient Health Questionnaire-9 (PHQ-9 $[54,55] ; \leq 10$ vs. $>10)$ using block randomisation of variable sizes will be performed.

\section{Blinding}

Participants will be blinded regarding study hypotheses but it will not be possible to blind them or study psychotherapists to allocation for obvious reasons. Furthermore, all variables at baseline will be collected prior to allocation to minimise reporting and selection bias.

\section{Data management and analysis \\ Data management}

An electronic case report form (eCRF) will be used for data collection. Data from self-reported questionnaires will be collected web-based electronically or paperbased. Paper-based data will be entered into the eCRF by a study assistant. Paper-and-pencil questionnaires will be stored in locked filing cabinets and electronic data will be stored on secured servers. To assure a safe and secure environment for data acquired, data transmission is encrypted with secure socket layer (SSL) technology. Only authorized users are able to enter or edit data and access is restricted to data of the patients in the respective centre. All changes to data are logged with a computerized timestamp in an audit trail. To guarantee high data quality, data validation rules will be defined in a data validation plan. Completeness, validity and plausibility of data will be checked in time of data entry (edit-checks) and using validating programs, which will generate queries. The investigator or the designated representatives are obliged to clarify or explain the queries.

If no further corrections are to be made in the database, eCRF data will be locked. Data will finally be downloaded and used for statistical analysis. All data management procedures will be conducted according to written defined standard operating procedures that guarantee an efficient conduct complying with good clinical practice.

Except contact details of participants, all data will be pseudonymised. Contact details will be stored locally in the study centre in a separate document and are only accessible for authorised staff of the local study centre.

\section{Sample size calculations}

Sample size calculations were performed with PASS Version 16.0.3. Congruent with results of a recent uncontrolled trial, we expect that participation in psychotherapeutic consultation at work may reduce days of sickness absence from 36 days/ 6 months to 26 days $/ 6$ months at follow-up with a standard deviation of 35 days/6 months for both time points [73]. For the control 
Table 1 Overview on additional study variables

\begin{tabular}{|c|c|c|}
\hline Variable & Participants & Method \\
\hline \multicolumn{3}{|l|}{ Interview data } \\
\hline $\begin{array}{l}\text { Global Assessment of } \\
\text { Functioning }\end{array}$ & All & Global Assessment of Functioning Scale (GAF $[30,31])$ \\
\hline Diagnosis & All & Derived from International Neuropsychiatric Interviews (MINI [32]) \\
\hline Indication of treatment & All & \\
\hline Childhood trauma & All & Childhood trauma screener [51] \\
\hline Work-related diagnostics & Intervention group & E.g. work history, job description, work tasks, work relationships, person-job fit $[25,33]$ \\
\hline \multicolumn{3}{|l|}{ Questionnaire data } \\
\hline $\begin{array}{l}\text { Sociodemographic } \\
\text { variables I }\end{array}$ & All & Age, gender, work experience \\
\hline $\begin{array}{l}\text { Sociodemographic } \\
\text { variables II }\end{array}$ & All & $\begin{array}{l}\text { Marital status, profession, weekly working hours, shift work, leadership position, company size, } \\
\text { professional perspective, physical illness }\end{array}$ \\
\hline Migration status & All & $\begin{array}{l}\text { Basic set of indicators for mapping migrant status; German nationality, parental German } \\
\text { nationality [52] }\end{array}$ \\
\hline Demands of immigration & $\begin{array}{l}\text { Participants with } \\
\text { migrant background }\end{array}$ & Demands of Immigration Scale (DIS [53] \\
\hline $\begin{array}{l}\text { Mental health - } \\
\text { depression }\end{array}$ & All & Depression items of the Patient-Health Questionnaire (PHQ-9 [54-56]) \\
\hline Mental health - anxiety & All & Generalized Anxiety Disorders (GAD-2 [57]) \\
\hline $\begin{array}{l}\text { Mental health - } \\
\text { somatoform disorders }\end{array}$ & All & Somatic Symptom Scale 8 (SSS-8 [58]) \\
\hline General health status & All & General health status item of the Veterans RAND 12 (VR-12 [59]) \\
\hline Cultural adaptation & $\begin{array}{l}\text { Participants with } \\
\text { migrant background }\end{array}$ & Frankfurt acculturation scale (FRAKK $[60,61])$ \\
\hline $\begin{array}{l}\text { Work productivity and } \\
\text { activity impairment }\end{array}$ & All & Work productivity and activity impairment Questionnaire (WPAI [62]) \\
\hline Subjective work ability & All & First item of the Work ability index (WAI $[63,64])$ \\
\hline Work role functioning & All & Work Role Functioning Questionnaire (WRPQ 2.0 [65]) - short version \\
\hline $\begin{array}{l}\text { Psychosocial working } \\
\text { conditions }\end{array}$ & All & $\begin{array}{l}\text { Third version of the Copenhagen Psychosocial Questionnaire (COPSOQ }[66,67]) \text { including } \\
\text { scales of job demands, control, development opportunities and social support }\end{array}$ \\
\hline $\begin{array}{l}\text { Psychosocial safety } \\
\text { climate }\end{array}$ & All & Short version of the Psychosocial Safety Climate Questionnaire [68] \\
\hline Personality Functioning & All & $\begin{array}{l}\text { German version of the Level of Personality Functioning-screener brief form (LPFS-BF } 2.0 \text { [69, } \\
\text { 70]) }\end{array}$ \\
\hline Integrated care & All & $\begin{array}{l}\text { Modified version of a questionnaire on experiences on integrated care including scales of } \\
\text { general coordination and coordination within and between care teams [71] }\end{array}$ \\
\hline
\end{tabular}

group, we expect that days of sickness absence will increase from 36 days $/ 6$ months to 42 days/ 6 months due to the lack of work-related treatment in CAU [74] and possibly deterioration of health. The same standard deviation of 35 days/ 6 months will be expected for the control group. A negative binominal distribution of days of sickness absence will be expected in accordance with previous research [73]. Following those expectations, a dispersion parameter of 2.12 was calculated. To detect this difference with a power of $80 \%$ and assuming a twotailed level of significance of $5 \%$, a sample size of 310 participants with 155 participants for each group is required. Assuming dropout rates of 40\% [15], 520 participants shall be recruited for the study in equal proportions per study centre.

\section{Statistical methods}

The treatment effect on the primary outcome (days of sickness absence within the last 6 months at the second follow-up) will be analysed using a mixed negative binomial regression model. Treatment group, days of sickness absence within the last 6 months before baseline, gender, age, subjective work ability at baseline as well as the centre will be included as covariates and the respective company as random effect. The primary analysis will be conducted based on the full analysis set according to 
Table 2 Schedule of the study

\begin{tabular}{|c|c|c|c|}
\hline Timepoint & $\begin{array}{c}\text { Baseline } \\
\mathrm{t}_{0}\end{array}$ & $\begin{array}{c}1^{\text {st }} \text { Follow-up } \\
t_{1} \\
\text { (9 months } \\
\text { after } t_{0)}\end{array}$ & $\begin{array}{c}2^{\text {nd }} \text { Follow up } \\
t_{2} \\
\text { (15 months } \\
\left.\text { after } t_{0}\right)\end{array}$ \\
\hline \multicolumn{4}{|l|}{ Enrolment } \\
\hline - Screening of eligibility & $x$ & & \\
\hline - Informed consent & $x$ & & \\
\hline $\begin{array}{l}\text { - Randomisation } \\
\text { Intervention }\end{array}$ & $x$ & & \\
\hline - Module 1-A & $\mathrm{x}$ & & \\
\hline - Module 1-B, 2-A, 2-B, 3 & $\leftarrow$ & $\rightarrow$ & \\
\hline \multicolumn{4}{|l|}{ Assessments } \\
\hline - Days of sickness absence & $x$ & $x$ & $x$ \\
\hline - Self-efficacy & $x$ & $x$ & $x$ \\
\hline - Quality of life & $x$ & $x$ & $x$ \\
\hline - Medical expenses & $x$ & $x$ & $x$ \\
\hline - Sociodemographic variables I & $x$ & & \\
\hline - Sociodemographic variables II & $x$ & $x$ & $x$ \\
\hline - Migration status & $\mathrm{x}$ & & \\
\hline - Demands of immigration & $x$ & $x$ & \\
\hline - Mental health & $x$ & $x$ & $x$ \\
\hline - General health status & $x$ & $x$ & $x$ \\
\hline - Childhood trauma & $x$ & & \\
\hline - Cultural adaptation & $x$ & & \\
\hline - Work productivity and activity impairment & $\mathrm{x}$ & $x$ & $x$ \\
\hline - Subjective work ability & $x$ & $x$ & $x$ \\
\hline - Work role functioning & $x$ & & $x$ \\
\hline - Psychosocial working conditions & $x$ & & \\
\hline - Psychosocial safety climate & $x$ & & \\
\hline - Personality functioning & $\mathrm{x}$ & & \\
\hline - Integrated care & & $x$ & \\
\hline - Global assessment of functioning & $\mathrm{x}$ & & \\
\hline - Diagnosis & $x$ & & \\
\hline - Indication of treatment & $x$ & & \\
\hline - Work-related diagnostics ${ }^{1}$ & $x$ & & \\
\hline
\end{tabular}


the intention-to-treat (ITT) principle (i.e. all patients will be analysed in the group they were randomised to).

Missing data for the primary and secondary outcome analysis is assumed to be "missing at random" and replaced using multiple imputation based on predictive mean matching using the covariates of the primary and secondary outcome analysis as potential predictors [75].

As a sensitivity analysis, an evaluation based on the per-protocol (PP) population will be performed. The PP set consists of all participants without major protocol violations. For this analysis, no imputation of missing data will be performed.

In case of significance of the ITT analysis of the primary outcome (two-sided confidence level of 5\%), a hierarchical linear mixed model will be used to analyse treatment effects on self-efficacy. This model will include treatment group, self-efficacy at baseline, gender, age, subjective work ability at baseline as well as the centre as covariates and company as random effect. Applying this hierarchical testing strategy, the overall type I error rate will still be controlled.

If no significance of the ITT analysis is achieved, the results of this analysis will be interpreted only in a descriptive sense.

Furthermore, subgroup analyses will be performed to evaluate the role of additional variables (e.g. indication of treatment, type of treatment, symptom severity) on treatment effects on the primary and secondary outcome.

Further collected data (see Table 1) will be analysed using appropriate descriptive methods.

Details of the statistical analysis will be further determined in a statistical analysis plan which will be written before database closure.

All those analyses will be conducted using SAS 9.4 or higher.

For the health economic evaluation, stochastic uncertainty of ICUR will be estimated by means of nonparametric bootstrapping with 2000 replications [76]. ICUR will be interpreted based on the cost-effectiveness plan [43]. MWTP necessary for the gain of one QALY by the implementation of the intervention in comparison to CAU will be estimated for the threshold range between 0 and 125.000€ [77] based on the cost effectiveness acceptability curve $[43,44,76]$. The health economic evaluation will be conducted using STATA 16.

\section{Monitoring}

\section{Data monitoring}

An independent data monitoring committee (DMC) will be established composed of two clinical professionals and one biometric professional. The DMC will be regularly informed about the course of the trial and all safety issues. Furthermore, the DMC will be asked for advice whether to continue, modify or stop the trial.

\section{Harms}

Occurrence of adverse events will be documented. Although adverse events are rare during psychotherapy [78], they cannot be completely ruled out. Adverse events may include lack of treatment results, occurrence of new symptoms, increasing symptoms, strains in therapist-patient relationship, strains or changes in work, family or other social relationships, stigmatisation and development of pathological dependency to the therapist [79-81]. In case of adverse events, the psychotherapist and possibly supervisors will weight up further treatment needs as described under Discontinuation and Modifications of the intervention protocol. Furthermore, measures will be taken to avoid adverse events including i) the educational programme for psychotherapists to increase awareness of adverse events, ii) supervision of psychotherapists and iii) progress monitoring as described by Rozental [82].

\section{Formative evaluation}

A formative evaluation of the intervention using qualitative methods will be realised. Data will be collected in focus groups and individual interviews. The focus groups and interviews will be recorded and transcribed.

First, prior to the intervention, two focus groups (approximately eight participants per group) are conducted with a) health care professionals and b) employees who have experience with psychotherapeutic consultation and return to work. These focus groups will be analysed using qualitative content analysis with regard to expectations of the participants, facilitating and hindering factors related to early prevention in company settings and future implementation of the intervention.

Second, four narrative focus groups (approximately eight participants per group) will be conducted with a) organisational and $b$ ) external experts in the RTW process before and after the implementation of the intervention. The focus groups will be analysed using qualitative content analysis. Expectations of the intervention, facilitating and hindering factors as well as valuation of the intervention will be examined. In addition, knowledge and handling of mental illness will be reconstructed.

Third, a subsample of 20 participants of the intervention group will be questioned in individual narrative interviews at two time points: at the end of the intervention and approximately 6 months later after (gradual) RTW. In more detail, the following subsamples of participants will be interviewed:

- Up to six participants who only received module 2A and/or 3

- Up to five participants who received module 2-A as well as outpatient or in patient acute treatment and module 3 
- Up to five participants who received module 2-A, vocational rehabilitation as well as module 3

- Up to four participants who only received module 2B.

In addition, five participants of the control group will be narratively interviewed 6 months after returning to work. All individual interviews will be analysed using the Documentary Method of Interpretation [83]. This method not only allows to identify reflexive knowledge, but above all to reconstruct tacit knowledge, knowledge of action and experiences. Participants' expectations of the intervention, experiences and behaviour in the process of intervention, as well as the effect of the intervention on RTW will be reconstructed. In addition, interactions between intervention modules and contextual conditions as well as facilitating and hindering factors will be analysed. Furthermore, effects of the intervention on self-management and self-efficacy as well as the valuation of the intervention by the participants will be examined.

Fourth, based on an overarching case comparison, similarities and differences will be identified in focus groups and individual interviews (referring to the interest in knowledge as mentioned above). The comparison allows evaluation of the intervention beyond the individual case. In summary, the formative evaluation aims to identify facilitating and hindering factors concerning the various intervention modules and in relation to different contextual conditions such as company size and structure.

\section{Discussion}

Worldwide, a treatment gap for CMDs has been described $[5,6]$. Inadequate and late treatment may increase the risk of chronicity of CMDs [84] and therefore might contribute to long and recurrent sickness absence and premature retirement $[3,4]$. The workplace and its working conditions may contribute beneficially or adversely to the development of CMDs [85-87]. At the same time, the workplace might function as a setting to enable utilization of mental health care interventions by reaching a high number of individuals suffering from CMDs [12].

This study protocol therefore describes a multicentre RCT, which aims to test psychotherapeutic consultation at work. In this study, psychotherapeutic consultation at work consists of a tailored, module-based and workrelated psychotherapy. It especially aims to reach patients at early disease stages as well as patients with subclinical symptoms [17]. Due to long waiting times for psychotherapeutic offers in the German health care system [10], we expect that patients of the control group are treated at later time points. We therefore expect that they also report more days of sickness absence than patients of the intervention group. Besides disorderspecific psychotherapy, the intervention group will also receive work-related psychotherapy and elaborate cooperation between mental health care actors. Consistent with previous research $[19,20,22,37,88]$, those aspects may result in additional reduction of sickness absence in the intervention group. In case that the intervention is shown to be effective to reduce sickness absence and referring to the high prevalence and burden of disease of CMDs [1, 2], implementation of psychotherapeutic consultation at work into practice could have large public health relevance.

If the intervention is shown to be effective to reduce days of sickness absence, we expect that direct and indirect costs related to CMDs are reduced. An additional health economic evaluation will therefore be performed to test whether the intervention is actually cost effective. Previous health economic evaluations of similar interventions $[16,20]$ may raise expectations of a positive evaluation. In this case, implementation of psychotherapeutic consultation at work might be of economic relevance by relieving financial pressure on health and pension insurances and reducing costs related to loss of productivity due to CMDs.

In Germany, large-scale implementation of psychosomatic consultation at work might be realized by joint financing by health and pension insurances. In accordance with German Social Law, module 1 and 2 might be financed by health insurances as special forms of health care ( $\$ 140$ a SGB V) and module 3 might be financed by the German pension insurance as employment participation benefits ( $\$ 49$ SGB IX). Besides publication of research results in international research journals, a manual of the intervention will be developed and made available to the public to support implementation of psychotherapeutic consultation at work into practice.

\section{Strengths and limitations}

Strengths of this study will include the randomised controlled study design, the large sample size and recruitment of employees from various companies. By including small, middle and large-sized companies in public and private industry around five study centres in Germany, we aim to improve external validity of our study results. However, external validity outside Germany will be limited due to differences in health care systems.

The fact that the control group will receive a first session of psychotherapeutic consultation at work including basic clinical assessment and recommendations for CAU within the German health care system might be perceived as a second limitation of this study. With this approach, treatment of the control group does not 
completely resemble CAU. Psychosomatic consultation at work has been shown to reach patients at earlier disease stages than CAU [12]. The control group could consequently receive earlier diagnoses and due to recommendations could also receive earlier treatment in CAU compared to usual study-independent conditions. This may further lead to faster recovery, better prognosis, prevention of chronicity $[84,89]$ and subsequently to reduced days of sickness absence. Intervention effects could therefore be underestimated. However, this approach is chosen for practical and ethical reasons. First, eligibility to participate in the study will be evaluated during basic diagnostics. Second, acceptance of the study by companies and their employees is thought to be increased by the fact that each study participant will receive at least one session of psychotherapeutic consultation at work. Third, giving recommendations for further treatment after diagnosis of health issues should be self-evident for ethical reasons.

Recruitment of study participants might be complicated by stigmatisation of mental disorders $[7,8]$, which is especially present in workplace settings [90-92]. Employees might fear that their colleagues, supervisors and employers could find out about visits to psychotherapeutic consultation at work and might therefore reject study participation [93]. To prevent fear of being seen by colleagues and supervisors when visiting psychotherapeutic consultation at work, efforts will be made to provide consultation in secure and confidential facilities. Furthermore, confidentiality of study participation towards third parties will always be guaranteed.

\section{Conclusion}

This protocol describes a multicentre RCT to test intervention effects of a tailored and module-based type of psychotherapeutic consultation at work. By providing prevention, early treatment, work-related psychotherapy and inducing collaboration between key (mental) health care professionals, the interventions aims to reduce days of sickness absence and increase self-efficacy of employees with CMDs. Referring to the high prevalence of CMDs [1], their large burden of disease [2] and economic impact on labour markets and social security systems [94], a positive evaluation of the intervention and subsequent implementation into practice could be of large public health and economic relevance.

\footnotetext{
Abbreviations

CAU: Care as usual; CMD: Common mental disorder; CSSRI: Client Sociodemographic and Service Receipt Inventory; DMC: Data monitoring committee; ICD: International Statistical Classification of Diseases and Related Health Problems; ICUA: Incremental cost-utility analysis; ITT: Intention-to-treat; MWTP: Maximum willingness to pay; PP: Per-protocol; RCT: Randomised controlled trial; RTW: Return to work; RTW-SE: Return-to-work self-efficacy scale; QALY: Quality adjusted life year
}

\section{Supplementary Information}

The online version contains supplementary material available at https://doi. org/10.1186/s12889-021-11195-9.

Additional file 1. Study questionnaire for sociodemographic variables and days of sickness absence.

Additional file 2. SPIRIT 2013 checklist.

\section{Acknowledgements}

We would like to thank Dr. Ulrich Adam-Keßler from Reha-Zentrum Teltow, Dr. Hans-Peter Unger from Asklepios Hospital Harburg and Prof. Dr. Adrian Loerbroks from the Institute of Occupational-, Social- and Environmental Medicine of the Heinrich-Heine-University Düsseldorf for their help during study development.

\section{Authors' contributions}

All authors contributed to the design of the study with main contributors being HG, PA and ER. CKr was the main contributor in designing the study intervention. JW was the main contributor in drafting the manuscript and was significantly supported by KH, ER, RKr, CKI, MF, RKi, YE, UW, UBS and RS $\mathrm{KH}$ provided expertise to selection of study variables, modifications of the intervention protocol and monitoring. RKr, CKI and MF provided expertise on data monitoring, data management and statistical analyses. RKi provided expertise on the health economic evaluation. YE provided expertise on diversity issues. UW, UBS and RS were responsible for the design of the formative evaluation. All authors provided feedback on the initial draft of the manuscript and read and approved the final version.

\section{Funding}

The study is financed by the German Federal Ministry of Education and Research (BMBF) under the project number 01GX1902. The BMBF was not involved in the design of the study. They will further not be involved in data collection, data management, statistical analyses and publishing study results. Open Access funding enabled and organized by Projekt DEAL.

\section{Availability of data and materials \\ Not applicable.}

\section{Declarations}

Ethics approval and consent to participate

This study was approved by the ethics committees of Ulm University in November 2020 (Application No. 339/20), of Friedrich-Alexander University Erlangen-Nürnberg in December 2020 (Application No. 525_20 BC), of University of Hildesheim Foundation in January 2021 (Application No. 165) and of Heinrich-Heine-University Düsseldorf in January 2021 (Application No. 20211279). Prior to the first intervention session, participants will be informed about the study and will receive standardised participant information sheets. Then, voluntary written informed consent for study participation and storage, evaluation and transfer of study-related data will be obtained from each study participant by research associates of the respective study centre. Withdrawal of written consent is possible at any time, without giving reasons.

Consent for publication

Not applicable.

\section{Competing interests}

The authors declare that they have no competing interests.

\section{Author details}

${ }^{1}$ Institute of Occupational, Social and Environmental Medicine, Centre for Health and Society, Medical Faculty, Heinrich-Heine-University Düsseldorf, Moorenstraße 5, 40225 Düsseldorf, Germany. ${ }^{2}$ Research Group Psychosomatic Rehabilitation, Department of Psychosomatic Medicine, Center for Internal Medicine and Dermatology, Charité - Universitätsmedizin Berlin, corporate member of Freie Universität Berlin and Humboldt-Universität zu Berlin, Hindenburgdamm 30, 12203 Berlin, Germany. ${ }^{3}$ Institute of Medical Biometry and Informatics, University Hospital of Heidelberg, Im Neuenheimer Feld 130.3, 69120 Heidelberg, Germany. ${ }^{4}$ Department of Psychosomatic Medicine and Psychotherapy, University Hospital of Erlangen, Friedrich-Alexander 
University Erlangen-Nürnberg (FAU), Schwabachanlage 6, 91054 Erlangen, Germany. ${ }^{5}$ nstitute of Psychology, University of Hildesheim Foundation, Universitätsplatz 1, 31141 Hildesheim, Germany. ${ }^{6}$ Institute of Medical Biometry and Statistics, Section of Health Care Research and Rehabilitation Research, Faculty of Medicine and Medical Center, University of Freiburg, Hugstetter Straße 49, 79106 Freiburg, Germany. ${ }^{7}$ Department Psychiatry II, Section of Health Economics and Psychiatric Services Research, Ulm University, Lindenallee 2, 89312 Günzburg, Germany. ${ }^{8}$ Federal Institute for Occupational Safety and Health (BAuA) Division 3 Work and Health Unit 3.5 Evidence-based Occupational Health, Workplace Health Management, Nöldnerstr, 40-42 10317 Berlin, Germany. ${ }^{9}$ Department of Psychosomatic Medicine and Psychotherapy, Ulm University Medical Center,

Albert-Einstein-Allee 23, 89081 Ulm, Germany.

Received: 18 May 2021 Accepted: 3 June 2021

Published online: 22 June 2021

\section{References}

1. Steel Z, Marnane C, Iranpour C, Chey T, Jackson JW, Patel V, et al. The global prevalence of common mental disorders: a systematic review and metaanalysis 1980-2013. Int J Epidemiol. 2014;43(2):476-93. https://doi.org/10.1 093/ije/dyu038.

2. Wittchen HU, Jacobi F, Rehm J, Gustavsson A, Svensson M, Jönsson B, et al. The size and burden of mental disorders and other disorders of the brain in Europe 2010. Eur Neuropsychopharmacol. 2011;21(9):655-79. https://doi. org/10.1016/j.euroneuro.2011.07.018.

3. Marschall J, Hildebrandt S, Nolting H-D. DAK Gesundheitsreport 2019. Hamburg: DAK; 2019.

4. Amiri S, Behnezhad S. Depression and risk of disability pension: A systematic review and meta-analysis. Int J Psychiatry Med. 2019:91217419837412. https://doi.org/10.1177/0091217419837412. Epub ahead of print.

5. Alonso J, Liu Z, Evans-Lacko S, Sadikova E, Sampson N, Chatterji S, et al. Treatment gap for anxiety disorders is global: results of the world mental health surveys in 21 countries. Depress Anxiety. 2018;35(3):195-208. https:// doi.org/10.1002/da.22711.

6. Thornicroft G, Chatterji S, Evans-Lacko S, Gruber M, Sampson N, AguilarGaxiola S, et al. Undertreatment of people with major depressive disorder in 21 countries. Br J Psychiatry. 2017;210(2):119-24. https://doi.org/10.1192/bjp. bp.116.188078.

7. Schomerus G, Stolzenburg S, Freitag S, Speerforck S, Janowitz D, EvansLacko S, et al. Stigma as a barrier to recognizing personal mental illness and seeking help: a prospective study among untreated persons with mental illness. Eur Arch Psychiatry Clin Neurosci. 2019;269(4):469-79. https://doi. org/10.1007/s00406-018-0896-0.

8. Clement S, Schauman O, Graham T, Maggioni F, Evans-Lacko S, Bezborodovs $\mathrm{N}$, et al. What is the impact of mental health-related stigma on help-seeking? A systematic review of quantitative and qualitative studies. Psychol Med. 2015;45(1):11-27. https://doi.org/10.1017/S0033291714000129.

9. Westin AML, Barksdale CL, Stephan SH. The effect of waiting time on youth engagement to evidence based treatments. Community Ment Health J. 2014;50(2):221-8. https://doi.org/10.1007/s10597-012-9585-z.

10. Bundespsychotherapeutenkammer. Ein Jahr nach der Reform der Psychotherapie-Richtlinie: Wartezeiten 2018. Berlin: BPtK; 2018.

11. Trusler K, Doherty C, Mullin T, Grant S, McBride J. Waiting times for primary care psychological therapy and counselling services. Couns Psychother Res. 2006;6(1):23-32. https://doi.org/10.1080/14733140600581358.

12. Rothermund E, Kilian R, Rottler E, Mayer D, Hölzer M, Rieger MA, et al. Improving access to mental health care by delivering psychotherapeutic Care in the Workplace: a cross-sectional exploratory trial. PLoS One. 2017; 12(1):e0169559. https://doi.org/10.1371/journal.pone.0169559.

13. Bode $K$, Wunsch E-M, Finger F, Kröger C. Interdisziplinäre Versorgung von Arbeitnehmern mit psychischen Störungen: Ein Faktencheck am Beispiel des Salzgitter-Modells. Psychother Psychosom Med Psychol. 2016;66(06): 235-41.

14. Preiser C, Wittich A. Rieger MA: [psychosomatic consultation in the workplace--description and modelling of a new health-related service]. Gesundheitswesen. 2015;77(11):e166-71. https://doi.org/10.1055/s0034-1384611.

15. Rothermund $E$, Gündel $H$, Rottler $E$, Hölzer M, Mayer D, Rieger M, et al. Effectiveness of psychotherapeutic consultation in the workplace: a controlled observational trial. BMC Public Health. 2016;16(1):891. https://doi. org/10.1186/s12889-016-3567-y.

16. Evans-Lacko S, Koeser L, Knapp M, Longhitano C, Zohar J, Kuhn K. Evaluating the economic impact of screening and treatment for depression in the workplace. Eur Neuropsychopharmacol. 2016;26(6):1004-13. https:// doi.org/10.1016/j.euroneuro.2016.03.005.

17. Preiser C, Rothermund E, Wittich A, Gundel H, Rieger MA. Psychosomatic consultation in the workplace: opportunities and limitations of the services offered-results of a qualitative study. Int Arch Occup Environ Health. 2016; 89(4):599-608. https://doi.org/10.1007/s00420-015-1098-y.

18. Rothermund E, Michaelis M, Jarczok MN, Balint EM, Lange R, Zipfel S, et al. Prevention of Common Mental Disorders in Employees. Perspectives on Collaboration from Three Health Care Professions. Int J Environ Res Public Health. 2018;15(2):278.

19. Reifferscheid A, Wege N, Riesbeck M, Janssen B. Rückkehr an den Arbeitsplatz nach depressiver Erkrankung - Evaluation einer retrospektiven Fall-Kontroll-Studie eines betriebsnahen integrierten Versorgungsmodells. Fortschr Neurol Psychiatr. 2019;87(2):121-7. https://doi.org/10.1055/s0043-124593.

20. Schene AH, Koeter MW, Kikkert MJ, Swinkels JA, McCrone P. Adjuvant occupational therapy for work-related major depression works: randomized trial including economic evaluation. Psychol Med. 2007;37(3):351-62. https:// doi.org/10.1017/S0033291706009366.

21. Hees HL, de Vries G, Koeter MWJ, Schene AH. Adjuvant occupational therapy improves long-term depression recovery and return-to-work in good health in sick-listed employees with major depression: results of a randomised controlled trial. Occup Environ Med. 2013;70(4):252-60. https:// doi.org/10.1136/oemed-2012-100789.

22. Gismervik S, Aasdahl L, Vasseljen O, Fors EA, Rise MB, Johnsen R, et al. Inpatient multimodal occupational rehabilitation reduces sickness absence among individuals with musculoskeletal and common mental health disorders: a randomized clinical trial. Scand J Work Environ Health. 2020; 46(4):364-72. https://doi.org/10.5271/sjweh.3882.

23. Øverland S, Grasdal AL, Reme SE. Long-term effects on income and sickness benefits after work-focused cognitive-behavioural therapy and individual job support: a pragmatic, multicentre, randomised controlled trial. Occup Environ Med. 2018;75(10):703-8. https://doi.org/10.1136/ oemed-2018-105137.

24. Lagerveld SE, Blonk RW, Brenninkmeijer V, Wijngaards-de Meij L, Schaufeli WB. Work-focused treatment of common mental disorders and return to work: a comparative outcome study. J Occup Health Psychol. 2012;17(2): 220-34. https://doi.org/10.1037/a0027049.

25. Kröger C, Bode K, Wunsch EM, Kliem S, Grocholewski A, Finger F. Workrelated treatment for major depressive disorder and incapacity to work: preliminary findings of a controlled, matched study. J Occup Health Psychol. 2015;20(2):248-58. https://doi.org/10.1037/a0038341.

26. Reme SE, Grasdal AL, Løvvik C, Lie SA, Øverland S. Work-focused cognitivebehavioural therapy and individual job support to increase work participation in common mental disorders: a randomised controlled multicentre trial. Occup Environ Med. 2015;72(10):745-52. https://doi.org/1 0.1136/oemed-2014-102700.

27. Niedermoser DW, Kalak N, Kiyhankhadiv A, Brand S, Walter C, Schweinfurth $\mathrm{N}$, et al. Workplace-related interpersonal group psychotherapy to improve life at work in individuals with major depressive disorders: a randomized interventional pilot study. Front Psychiatry. 2020;11:168. https://doi.org/10.33 89/fpsyt.2020.00168.

28. Schramm E, Mack S, Thiel N, Jenkner C, Elsaesser M, Fangmeier T. Interpersonal psychotherapy vs. Treatment as Usual for Major Depression Related to Work Stress: A Pilot Randomized Controlled Study. Front Psychiatry. 2020;11:193.

29. World Health Organization. ICD 10: international statistical classification of diseases and related health problems: tenth revision. 2nd ed. Geneva: World Health Organization; 2004.

30. Hall RC. Global assessment of functioning. A modified scale. Psychosomatics. 1995;36(3):267-75. https://doi.org/10.1016/S0033-3182 (95)71666-8.

31. Saß H, Wittchen HU, Zaudig M, Houben I. Diagnostische Kriterien des Diagnostischen und Statistischen Manuals Psychischer Störungen. DSM-IVTR. Göttingen: Hogrefe; 2003.

32. Sheehan DV, Lecrubier $Y$, Sheehan KH, Amorim P, Janavs J, Weiller $E$, et al. The Mini-International Neuropsychiatric Interview (M.I.N.I.): the development 
and validation of a structured diagnostic psychiatric interview for DSM-IV and ICD-10. J Clin Psychiatry. 1998;59(Suppl 20):22-33 quiz 34-57.

33. Bode K, Maurer F, Kröger C. Arbeitswelt und psychische Störungen. Göttingen: Hogrefe; 2017. https://doi.org/10.1026/02758-000.

34. Köllner V. Positionspapier der DGPPR zur Medizinisch-beruflich orientierten Rehabilitation (MBOR) in der Psychosomatik. Ärztliche Psychosomatik. 2018; 13:210-12.

35. Stegmann R, Schröder UB. Anders Gesund - Psychische Krisen in der Arbeitswelt. Prävention, Return-to-Work und Eingliederungsmanagement. Wiesbaden: Springer; 2018. https://doi.org/10.1007/978-3-658-17882-6.

36. Erim Y, Toker M, Aygün S, Özdemir Z, Renz M, Gün AK. Essener Leitlinien zur interkulturellen Psychotherapie. PiD. 2010;11(04):299-305. https://doi.org/1 0.1055/s-0030-1248638

37. Nieuwenhuijsen K, Faber B, Verbeek JH, Neumeyer-Gromen A, Hees HL, Verhoeven AC, et al. Interventions to improve return to work in depressed people. Cochrane Database Syst Rev. 2014;12:Cd006237.

38. Victor M, Lau B, Ruud T. Predictors of return to work among patients in treatment for common mental disorders: a pre-post study. BMC Public Health. 2017;18(1):27. https://doi.org/10.1186/s12889-017-4581-4.

39. Nigatu YT, Liu Y, Uppal M, McKinney S, Gillis K, Rao S, et al. Prognostic factors for return to work of employees with common mental disorders: a meta-analysis of cohort studies. Soc Psychiatry Psychiatr Epidemiol. 2017; 52(10):1205-15. https://doi.org/10.1007/s00127-017-1402-0.

40. Volker D, Zijlstra-Vlasveld MC, Brouwers EP, van Lomwel AG, van der FeltzCornelis CM. Return-to-work self-efficacy and actual return to work among long-term sick-listed employees. J Occup Rehabil. 2015;25(2):423-31. https:// doi.org/10.1007/s10926-014-9552-3.

41. Lagerveld SE, Blonk RWB, Brenninkmeijer V, Schaufeli WB. Return to work among employees with mental health problems: development and validation of a self-efficacy questionnaire. Work Stress. 2010;24(4):359-75. https://doi.org/10.1080/02678373.2010.532644.

42. Rigotti T, Schyns B, Mohr G. A short version of the occupational self-efficacy scale: structural and construct validity across five countries. J Career Assess. 2008;16(2):238-55. https://doi.org/10.1177/1069072707305763.

43. Glick H, Doshi JA, Sonnad SS. Economic evaluation in clinical trials. 2nd ed. Oxford: Oxford University Press; 2014.

44. Salize H, Kilian R. Gesundheitsökonomie in der Psychiatrie. Konzepte, Methoden, Analysen. Stuttgart: Kohlhammer; 2010.

45. Husereau D, Drummond M, Petrou S, Carswell C, Moher D, Greenberg D, et al. Consolidated health economic evaluation reporting standards (CHEE RS) statement. Value Health. 2013;16(2):e1-5. https://doi.org/10.1016/j.jval.2 013.02.010.

46. Chisholm D, Knapp MR, Knudsen HC, Amaddeo F, Gaite L, van Wijngaarden B. Client Socio-Demographic and Service Receipt Inventory--European Version: development of an instrument for international research. EPSILON Study 5. European Psychiatric Services: Inputs Linked to Outcome Domains and Needs. Br J Psychiatry Suppl. 2000;39:s28-33.

47. Roick C, Kilian R, Matschinger H, Bernert S, Mory C, Angermeyer MC. Die deutsche Version des Client Sociodemographic and Service Receipt Inventory. Psychiatr Prax. 2001;28(Sup. 2):84-90.

48. Herdman M, Gudex C, Lloyd A, Janssen M, Kind P, Parkin D, et al. Development and preliminary testing of the new five-level version of EQ-5D (EQ-5D-5L). Qual Life Res. 2011;20(10):1727-36. https://doi.org/10.1007/s1113 6-011-9903-X.

49. Leidl R, Reitmeir P. An experience-based value set for the EQ-5D-5L in Germany. Value Health. 2017;20(8):1150-6. https://doi.org/10.1016/j.jval.2017.04.019.

50. Ludwig K. Graf von der Schulenburg JM, Greiner W: German value set for the EQ-5D-5L. Pharmacoeconomics. 2018;36(6):663-74. https://doi.org/10.1 007/s40273-018-0615-8.

51. Grabe HJ, Block A, Schmidt CO, Appel K, Driessen M, Wingenfeld K, et al. Childhood trauma screener Testmanual. Aachen: Psychometrikon; 2015.

52. Schenk L, Bau AM, Borde T, Butler J, Lampert T, Neuhauser H, et al. Mindestindikatorensatz zur Erfassung des Migrationsstatus - Empfehlungen für die epidemiologische Praxis. Bundesgesundheitsbl Gesundheitsforsch Gesundheitsschutz. 2006;49(9):853-60. https://doi.org/10.1007/s00103-006-0018-4.

53. Aroian KJ, Norris AE, Tran TV, Schappler-Morris N. Development and psychometric evaluation of the demands of immigration scale. J Nurs Meas. 1998;6(2):175-94. https://doi.org/10.1891/1061-3749.6.2.175.

54. Gräfe K, Zipfel S, Herzog W, Löwe B. Screening psychischer Störungen mit dem "Gesundheitsfragebogen für Patienten (PHQ-D)". Diagnostica. 2004; 50(4):171-81. https://doi.org/10.1026/0012-1924.50.4.171.
55. Löwe B, SR L, Zipfel S, Herzog W. Gesundheitsfragebogen für Patienten (PHQ D). Komplettversion und Kurzform. In: Testmappe mit Manual, Fragebögen, Schablonen, vol. 2. Karlsruhe: Pfizer; 2002.

56. Spitzer RL, Kroenke K, Williams JB. Validation and utility of a self-report version of PRIME-MD: the PHQ primary care study. Primary care evaluation of mental disorders. Patient health questionnaire. JAMA. 1999;282(18):173744. https://doi.org/10.1001/jama.282.18.1737.

57. Kroenke K, Spitzer RL, Williams JB, Monahan PO, Löwe B. Anxiety disorders in primary care: prevalence, impairment, comorbidity, and detection. Ann Intern Med. 2007;146(5):317-25. https://doi.org/10.7326/0003-4819-146-5-2 00703060-00004.

58. Gierk B, Kohlmann S, Kroenke K, Spangenberg L, Zenger M, Brähler E, et al. The somatic symptom scale-8 (SSS-8): a brief measure of somatic symptom burden. JAMA Intern Med. 2014;174(3):399-407. https://doi.org/10.1001/ja mainternmed.2013.12179.

59. Selim AJ, Rogers W, Fleishman JA, Qian SX, Fincke BG, Rothendler JA, et al. Updated U.S. population standard for the veterans RAND 12-item health survey (VR-12). Qual Life Res. 2009;18(1):43-52. https://doi.org/10.1007/s1113 6-008-9418-2.

60. Morawa E, Erim Y. Acculturation and depressive symptoms among Turkish immigrants in Germany. Int J Environ Res Public Health. 2014;11(9):9503-21. https://doi.org/10.3390/ijerph110909503.

61. Bongard S, Etzler S, Frankenberg E. FRAKK - Frankfurter Akkulturationsskala, vol. 1. Göttingen: Hogrefe; 2020.

62. Reilly MC, Zbrozek AS, Dukes EM. The validity and reproducibility of a work productivity and activity impairment instrument. Pharmacoeconomics. 1993; 4(5):353-65. https://doi.org/10.2165/00019053-199304050-00006.

63. Hasselhorn HM, Freude G. Der Work Ability Index - ein Leitfaden. Wirtschaftsverlag NW: Bremerhaven; 2007.

64. Tuomi K, IImarinen J, Jahkola A, Katajarinne L, Tulkki A. Work ability index. 2nd ed. Helsinki: Finnish Institute of Occupational Health; 1999.

65. Abma Fl, Bültmann U, Amick lii BC, Arends I, Dorland HF, Flach PA, et al. The work role functioning questionnaire $\mathrm{v} 2.0$ showed consistent factor structure across six working samples. J Occup Rehabil. 2018;28(3):465-74. https://doi. org/10.1007/s10926-017-9722-1.

66. Burr $H$, Berthelsen $H$, Moncada $S$, Nübling $M$, Dupret $E$, Demiral $Y$, et al. The third version of the Copenhagen psychosocial questionnaire. Saf Health Work. 2019;10(4):482-503. https://doi.org/10.1016/j.shaw.2019.10.002.

67. Nübling M, Stößel U, Hasselhorn HM, Michaelis M, Hofmann F. Methoden zur Erfassung psychischer Belastungen. Wirtschaftsverlag NW: Bremerhaven; 2005.

68. Hall GB, Dollard MF, Coward J. Psychosocial safety climate: development of the PSC-12. Int J Stress Manag. 2010;17(4):353-83. https://doi.org/10.1037/a 0021320.

69. Bach B, Hutsebaut J. Level of personality functioning scale-brief form 2.0: utility in capturing personality problems in psychiatric outpatients and incarcerated addicts. J Pers Assess. 2018;100(6):660-70. https://doi.org/10.1 080/00223891.2018.1428984

70. Weekers LC, Hutsebaut J, Kamphuis JH. The level of personality functioning scale-brief form 2.0: update of a brief instrument for assessing level of personality functioning. Personal Ment Health. 2019;13(1):3-14. https://doi. org/10.1002/pmh.1434.

71. Walker KO, Stewart AL, Grumbach K. Development of a survey instrument to measure patient experience of integrated care. BMC Health Serv Res. 2016;16(1):193. https://doi.org/10.1186/s12913-016-1437-z.

72. Kobel F, Morawa E, Erim Y. Effectiveness of Inpatient Psychotherapy for Patients With and Without Migratory Background: Do They Benefit Equally? Front Psychiatry. 2020;11:542.

73. Gantner M, Scheider J, Brandner S, Rottler E, Gündel H, von Wietersheim J. Effekte einer Psychosomatischen Betriebsambulanz auf die Arbeitsunfähigkeitszeiten der Teilnehmer - eine Analyse anhand von Sekundärdaten. In: Deutscher Kongress für Psychosomatische Medizin und Psychotherapie: 21.03.2018-24.03.2018 2018; Berlin; 2018.

74. Streibelt M, Brünger M. Wie viele arbeitsbezogene Leistungen bekommen Patienten mit besonderen beruflichen Problemlagen? Analyse einer repräsentativen indikationsübergreifenden Stichprobe von Rehabilitanden. Rehabilitation (Stuttg). 2014;53(06):369-75.

75. van Buuren S. Multiple imputation of discrete and continuous data by fully conditional specification. Stat Methods Med Res. 2007;16(3):219-42. https:// doi.org/10.1177/0962280206074463.

76. Willan AR, Briggs AH. Statistical analysis of cost-effectiveness data. Chichester: Wiley \& Sons; 2006. https://doi.org/10.1002/0470856289. 
77. Remme M, Martinez-Alvarez M, Vassall A. Cost-effectiveness thresholds in Global Health: taking a multisectoral perspective. Value Health. 2017;20(4): 699-704. https://doi.org/10.1016/j.jval.2016.11.009.

78. Cuijpers $P$, Reijnders M, Karyotaki $E$, de Wit $L$, Ebert DD. Negative effects of psychotherapies for adult depression: a meta-analysis of deterioration rates. J Affect Disord. 2018;239:138-45. https://doi.org/10.1016/j.jad.2018.05.050.

79. Abeling B, Müller A, Stephan M, Pollmann I, de Zwaan M. Negative Effekte von Psychotherapie: Häufigkeit und Korrelate in einer klinischen Stichprobe. Psychother Psychosom Med Psychol. 2018;68(09/10):428-36.

80. Berk M, Parker $G$. The elephant on the couch: side-effects of psychotherapy. Aust N Z J Psychiatry. 2009;43(9):787-94. https://doi.org/10.1080/0004 8670903107559

81. Linden M. How to define, find and classify side effects in psychotherapy: from unwanted events to adverse treatment reactions. Clin Psychol Psychother. 2013:20(4):286-96. https://doi.org/10.1002/cpp.1765.

82. Rozental A, Castonguay L, Dimidjian S, Lambert M, Shafran R, Andersson G, et al. Negative effects in psychotherapy: commentary and recommendations for future research and clinical practice. BJPsych Open. 2018;4(4):307-12. https://doi.org/10.1192/bjo.2018.42.

83. Bohnsack R. Rekonstruktive Sozialforschung. Einführung in Qualitative Methoden: Opladen \& Toronto, Verlag Barbara Budrich, UTB; 2014

84. Dell'Osso B, Glick ID, Baldwin DS, Altamura AC. Can long-term outcomes be improved by shortening the duration of untreated illness in psychiatric disorders? A Conceptual Framework. Psychopathology. 2013;46(1):14-21. https://doi.org/10.1159/000338608.

85. Bonde JP. Psychosocial factors at work and risk of depression: a systematic review of the epidemiological evidence. Occup Environ Med. 2008;65(7): 438-45. https://doi.org/10.1136/oem.2007.038430.

86. Rugulies R, Aust B, Madsen IE. Effort-reward imbalance at work and risk of depressive disorders. A systematic review and meta-analysis of prospective cohort studies. Scand J Work Environ Health. 2017;43(4):294-306. https://doi. org/10.5271/sjweh.3632.

87. Modini M, Joyce S, Mykletun A, Christensen H, Bryant RA, Mitchell PB, et al. The mental health benefits of employment: results of a systematic metareview. Australas Psychiatry. 2016;24(4):331-6. https://doi.org/10.1177/103 9856215618523

88. Cullen KL, Irvin E, Collie A, Clay F, Gensby U, Jennings PA, et al. Effectiveness of workplace interventions in return-to-work for musculoskeletal, painrelated and mental health conditions: an update of the evidence and messages for practitioners. J Occup Rehabil. 2018;28(1):1-15. https://doi. org/10.1007/s10926-016-9690-X.

89. Kraus C, Kadriu B, Lanzenberger R, Zarate CA Jr, Kasper S. Prognosis and improved outcomes in major depression: a review. Transl Psychiatry. 2019; 9(1):127. https://doi.org/10.1038/s41398-019-0460-3.

90. Hampson ME, Watt BD, Hicks RE. Impacts of stigma and discrimination in the workplace on people living with psychosis. BMC Psychiatry. 2020;20(1): 288. https://doi.org/10.1186/s12888-020-02614-z.

91. Hipes C, Lucas J, Phelan JC, White RC. The stigma of mental illness in the labor market. Soc Sci Res. 2016;56:16-25. https://doi.org/10.1016/j.ssresea rch.2015.12.001.

92. Stuart H. Stigma and work. Healthc Pap. 2004;5(2):100-11. https://doi.org/1 0.12927/hcpap..16829.

93. Walton L. Exploration of the attitudes of employees towards the provision of counselling within a profit-making organisation. Counsell Psychother Res J. 2003;3(1):65-71. https://doi.org/10.1080/14733140312331384658.

94. OECD EU. Health at a glance: Europe 2018. Paris: OECD Publishing; 2018

\section{Publisher's Note}

Springer Nature remains neutral with regard to jurisdictional claims in published maps and institutional affiliations.

Ready to submit your research? Choose BMC and benefit from:

- fast, convenient online submission

- thorough peer review by experienced researchers in your field

- rapid publication on acceptance

- support for research data, including large and complex data types

- gold Open Access which fosters wider collaboration and increased citations

- maximum visibility for your research: over $100 \mathrm{M}$ website views per year

At $\mathrm{BMC}$, research is always in progress.

Learn more biomedcentral.com/submissions 